\title{
Electron Microscope Study of the Martensite Transformation in an Ordered $\mathrm{Fe}_{3} \mathrm{Pt}$ Alloy*
}

\author{
By Tsugio 'Tadaki** and Ken'ichi Shimizu**
}

\begin{abstract}
The martensitic transformation in an ordered $\mathrm{Fe}_{3} \mathrm{Pt}$ alloy has been studied by means of electron microscopy. The $b c c$ martensite was found to have a superlattice which was expected to be formed by the Bain distortion from the $\mathrm{Cu}_{3} \mathrm{Au}$-type superlattice of $\gamma$ phase. A number of transformation twins on the $\{112\}$ type (not of the $\{211\}$ and $\{121\}$ types) plane were observed in the martensite. The habit plane of martensite plates and the orientation relationship between the martensite and austenite were consistent with those in the disordered Fe-Pt alloy of the same composition.

The domain structures in the ordered $\gamma$ phase were observed by using the dark field method, and it was clarified that the Ms temperature dropped rapidly according as the size of domains increased.
\end{abstract}

(Received September 16, 1969)

\section{Introduction}

Some years ago Wechsler et al. (1) and Bowles and Mackenzie ${ }^{(2)}$ proposed independently a phenomenological theory which enables us to predict the crystallographic features in martensite transformation, that is, the habit plane, orientation relationship and other geometrical features. The theory involves two intrinsic assumptions of a lattice correspondence and a lattice invariant shear. In the case of the $f c c$ to $b c c$ transformation, their assumptions are known to be the Bain correspondence and the slip or twinning shear on a particular plane in the martensite crystals, respectively.

Although the latter shear has been observed in many iron alloys and steels by means of electron microscopy ${ }^{(3)}$ (5), the former Bain correspondence or distortion has not been verified directly except for previous work on a high aluminum steel by Nishiyama et al. ${ }^{(6)}$ They, however, were compelled to assume the superlattice of the matrix $\gamma$ phase to be the $\mathrm{Cu}_{3} \mathrm{Au}$ type because the Ms temperature of the specimen used was very high and then the matrix $\gamma$ phase was not retained. On the other hand, an ordered $\mathrm{Fe}-\mathrm{Pt}$ alloy with 25 at $\% \mathrm{Pt}$ atoms has a $\mathrm{Ms}$ point below room temperature, and thus the superlattice structure of the $\mathrm{Fe}_{3} \mathrm{Pt} \gamma$ phase can be confirmed directly and compared with that of the martensite crystal. Such a comparison will bring us some information with respect to the Bain distortion, as carried out in the present experiment.

Recently, stacking faults have been observed in the ordered $\beta^{\prime}$ martensites in some $\mathrm{Cu}$ alloys. The martensites are formed from the $b c c$ matrix having the $\mathrm{CsCl}$ or

* This paper was read before the Spring Meeting of the Japan Inst itute of Metals, March, 1969, and published in J. Japan Inst. Metals, 34 (1970), 77.

** The Institute of Scientific and Industrial Research, Osaka University, Suita, Osaka, Japan.

(1) M.S. Wechsler, D.S. Lieberman and T.A. Read : Trans. AIME, 197 (1953), 1503.

(2) J. S. Bowles and J. K. Mackenzie : Acta Met., 2 (1954), 129, $138,224$.

(3) Z. Nishiyama and K. Shimizu : J. Electronmicroscopy, Japan, 4 (1956), 51.

(4) Z. Nishiyama and K. Shimizu : Acta Met., 7(1959), 432.

(5) P. M. Kelly and J. Nutting : Proc. Roy. Soc., 259 (1960), 45.

(6) Z. Nishiyama, K. Shimizu and M. Harada : J. Japan Inst. Metals, 33 (1969), 871.
$\mathrm{Fe}_{3} \mathrm{Al}$-type superlattice and appear to have a close relation with the $f c c$ lattice ${ }^{(7) \sim(9)}$. The $\mathrm{Fe}_{3} \mathrm{Pt}$ alloy examined in the present experiment is considered to exhibit ordered $f c c$ to ordered $b c c$ transformation. Therefore, in some sense, this transformation is inverse to that in the above $\mathrm{Cu}$ alloys. Thus, a question arises whether the $\mathrm{Fe}_{3} \mathrm{Pt}$ ordered martensite contains stacking faults as in the case of the $\mathrm{Cu}$ alloys or twin faults as in the case of ordinary disordered iron alloys. The present experiment was also carried out to answer this question.

\section{Specimens and Experimental Procedures}

The alloy with a nominal composition of $25 \mathrm{at} \% \mathrm{Pt}$ was prepared by melting $99.99 \%$ pure iron and $99.99 \%$ pure platinum in a vacuum induction furnace. The difference in weight was hardly observed before and after the melting, so no chemical analysis was made. The cast ingot was hot-forged at $800^{\circ} \mathrm{C}$ and homogenized for one week at $1200^{\circ} \mathrm{C}$ in vacuum. Then the ingot was rolled and annealed repeatedly to make plates of about $0.2 \mathrm{~mm}$ in thickness, from which disc-shaped specimens of $3 \mathrm{~mm}$ in diameter were finally obtained.

After annealing at $1000^{\circ} \mathrm{C}$ in an evacuated quartz capsule for $1 \mathrm{hr}$, the specimens were quenched into water at room temperature. Subsequently heat treatment for ordering was carried out by holding at $650^{\circ} \mathrm{C}$ for $0.5 \mathrm{hr}$, followed by water quenching, and then subzero-cooled into various temperatures to produce a suitable amount of martensites. To examine some relations between the $\mathrm{Ms}_{\mathrm{s}}$ temperature and the domain size in the austenite matrix, the annealing time at $650^{\circ} \mathrm{C}$ was changed from 0.5 to $24 \mathrm{hr}$.

Optical microscopy was, firstly, made to examine the surface relief associated with the $\gamma \rightarrow \alpha^{\prime}$ transformation. For transmission electron microscopy, foils were obtained by electropolishing in a mixed solution of $60 \%$ phosphoric

(7) Z. Nishiyama and S. Ka j̈wara : Trans. JIM, 3(1962), 127.

(8) S. Sato and K. Takezawa : Proceedings of the International Conference on the Strength of Melals and Alloys, Supplement to Trans. JIM, Vol. 9, (1968), p. 925.

(9) Z. Nishiyama, K. Shimizu and H. Morikawa : Trans. JIM, 9 (1968), 307 
acid and $40 \%$ sulfuric acid kept at $15^{\circ} \mathrm{C}$. The P.T.F.E. holder method was employed and the current density was $5 \sim 10 \mathrm{~A} / \mathrm{cm}^{2}$. The electron microscope used was Hitachi HU-11 D-1, operated at $100 \mathrm{kV}$.

\section{Experimental Results and Discussions}

\section{Optical microscopy}

Photo. 1 is an optical micrograph taken from the surface of a specimen which was electro-polished at room temperature and then subzero-cooled into $-196^{\circ} \mathrm{C}$. There can clearly be seen surface relief effects. The existence of the surface relief and its shape show clearly that the $\mathrm{Fe}_{3} \mathrm{Pt}$ alloy subjected to an ordering treatment at $650^{\circ} \mathrm{C}$ for $0.5 \mathrm{hr}$ exhibits the "martensitic" phase transformation below room temperature (about $-50^{\circ} \mathrm{C}$ as will be shown later).

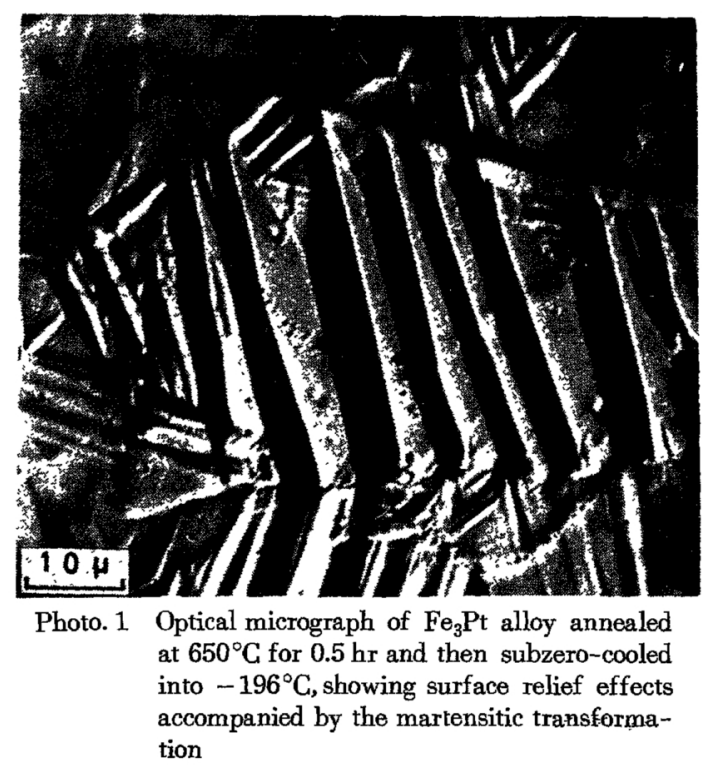

\section{Electron microscopy}

\section{(1) Superlattice structures of austenite and martensite}

Photo. 2 is a typical electron diffraction pattern taken from the matrix $\gamma$ phase of the (001) orientation. This shows clearly that the ordered structure of the $\mathrm{Fe}_{3} \mathrm{Pt} \gamma$ phase is of the $\mathrm{Cu}_{3} \mathrm{Au}$ type. This result is consistent with that obtained by Kussmann and Rittberg ${ }^{(10)}$ using an X-ray technique.

The Bain distortion for the $f c c$ to $b c c$ martensitic transformation is illustrated schematically in Fig. 1(a), the contraction axis being $[001]_{f}$. Since the matrix $\gamma$ phase has a superlattice of the $\mathrm{Cu}_{3} \mathrm{Au}$ type as described above, the $b c c$ martensite formed by the Bain distortion must have a definite superlattice as shown in Fig. 1 (b). In the figure, the parameter for the fundamental $b c c$ lattice is denoted by $a_{b}{ }^{*}$. The unit cell of the superlattice doubles in the $\mathrm{x}$-and $\mathrm{y}$-axes and the $\mathrm{Pt}$ atoms are situated at (000)

(10) A. Kussmann and G. G. V. Rittberg : Z. Metallk., 41 (1950), 470.

* Suffix $f$ and $b$ mean $f c c$ lattice of $\gamma$ phase and $b c c$ lattice of martensite phase, respectively.

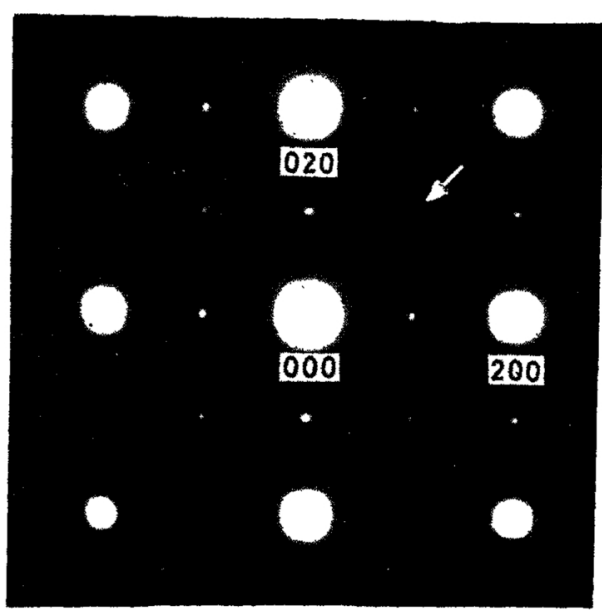

Photo. 2 Electron diffraction pattern taken from the retained $\gamma$ phase, showing the [001] zone of the $\mathrm{Cu}_{3} \mathrm{Au}$ type superlattice
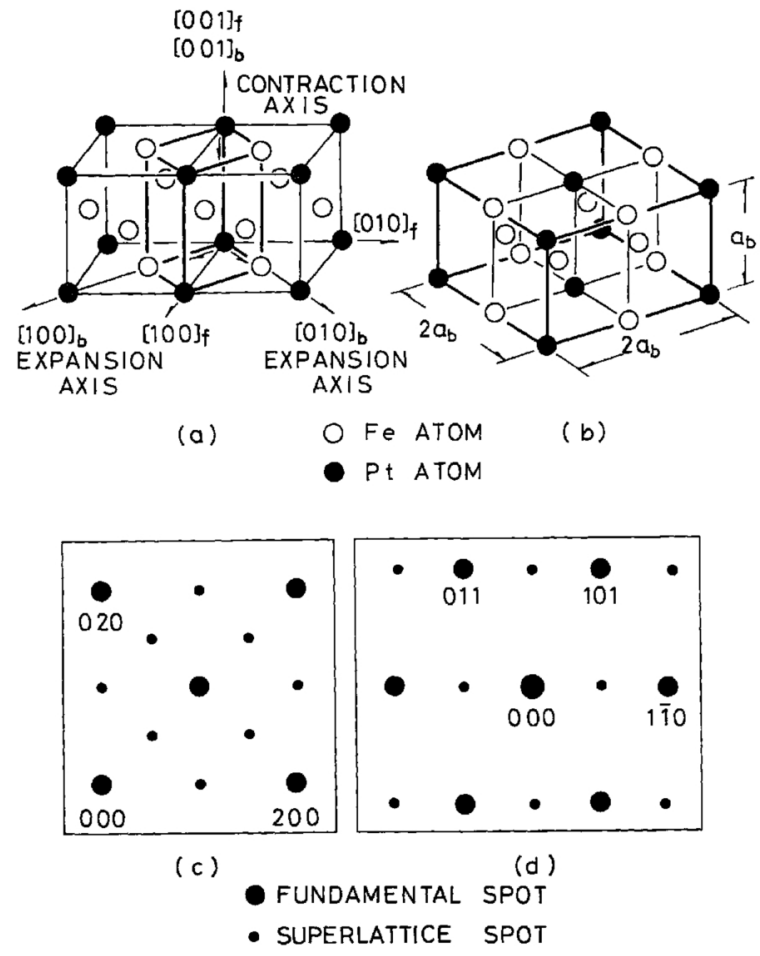

Fig. 1 (a) Bain correspondence and distortion for the $f c c$ to $b c c$ martensitic transformation

(b) An ordered unit cell in $b c c$ lattice formed by the Bain distortion.

(c) (d) (001) and (īi1) reciprocal lattice sections of the ordered $b c c$ lattice

and $(1 / 2,1 / 2,0)$. The structure factor of this lattice is the same as that given in the previous paper by Nishiyama et al., that is, the structure factor of the superlattice spot is

$$
F=F_{\text {atom }}\{1+\exp 2 \pi i(2 h+2 k) / 2\},
$$

where $F_{\text {atom }}$ is a factor containing the atomic scattering factors. The reciprocal lattice, thus, can be constructed easily and the (00I) and (III) reciprocal lattice sections with respect to the fundamental $b c c$ lattice are shown in Fig. 1 (c) and (d), respectively. In these figures, the reciprocal lattice points denoted with large closed circles are due to the fundamental $b c c$ lattice and those with 
small closed circles are due to the superlattice. Photo. $3(a)$ and $(b)$ are electron diffraction patterns taken from the martensite crystals and coincide with the above (001) and (1̄1) reciprocal lattice planes, respectively*. Thus, it can be concluded that the $b c c$ martensite in the $\mathrm{Fe}_{3} \mathrm{Pt}$ ordered alloy has a superlattice formed by the Bain distortion from the $\mathrm{Cu}_{3} \mathrm{Au}$ type superlattice of the $\gamma$ phase.
Photo. 4(a) is a typical electron micrograph showing the internal structures of a martensite crystal and (b) is the diffraction pattern taken from the area of (a). (a) reveals a number of parallel bands and the trace of bands is perpendicular to the direction of streaks in $(b)^{* *}$. The pattern (b), consists of three sets of reflections, which were identified as $\langle 310\rangle$, $\langle 211\rangle$ and $\langle 010\rangle$ zone

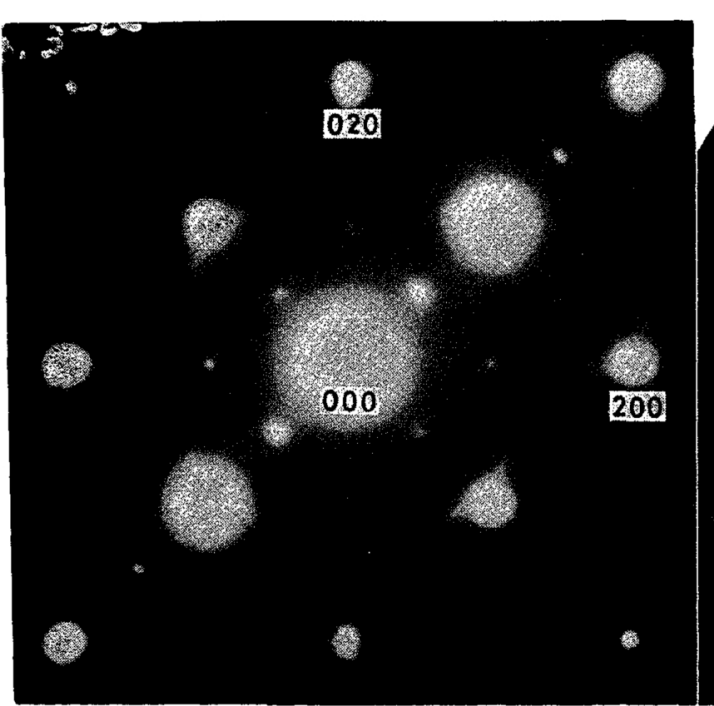

(a)

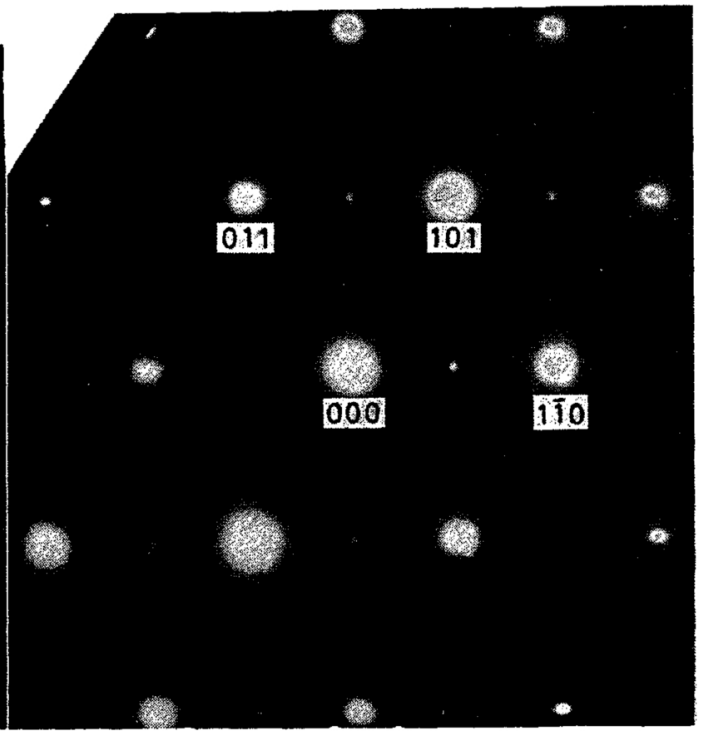

(b)

Photo. 3 Electron diffraction pattern taken from the $\mathrm{Fe}_{3} \mathrm{Pt}$ martensites, showing the [001] zone (a) and the [1111] zone (b)

In the previous experiment on $\mathrm{Fe}-\mathrm{Al}-\mathrm{C}$ alloy, the (100) and $(010)$ superlattice spots of the martensite crystal were very weak and were scarcely recognized in some cases. In the present experiment, however, such a intensity anomaly was not recognized in the diffraction patterns from the $\mathrm{Fe}_{3} \mathrm{Pt}$ martensite, Photos. 3 (a) and (b). This means that the behavior of the lattice invariant shear in the $\mathrm{Fe}_{3} \mathrm{Pt}$ martensite may be different from that in the $\mathrm{Fe}-\mathrm{Al}-\mathrm{C}$ martensite. In fact, electron micrographs of the former martensite have revealed mainly twin faults as will be shown later, while those of the latter martensite seemed to reveal a number of stacking faults in addition to twin faults. These stacking faults can be regarded as an origin of the intensity anomaly of $\{100\}$ reflections.

Now, some of the "out-of-step" structures in martensites can be inherited from those with the $1 / 2<110\rangle$ vector in the matrix $\gamma$ phase by the Bain distortion. Domain structures due to the "out-of-step" were surely observed in the dark-field images of the matrix $\gamma$ phase as will be shown later, and the intensity anomaly of $\{110\}$ superlattice spots was slightly recognized in the diffraction pattern, Photo. 2, as represented by an arrow. However, the "out-of-step" structures inherited into the martensite do not bring any remarkable anomaly in the intensity of $\{100\}$ reflections.

\section{(2) Internal structures}

* The lattice parameters of austenite and martensite in the present alloy were determined by electron diffraction, with the following result : $a_{f}=3.72 \AA$ and $a_{b}=2.96 \AA$, respectively. For the disordered alloy of the same composition, the parameters had been obtained by X-ray, which were $a_{f}=3.725 \AA$ and $a_{b}=2.967 \AA^{(11)}$. patterns, respectively, as indicated with three lines. All the variants of these zones are not equivalent since the martensite crystal has an ordered structure as shown in Fig. 1 (b). Let us now consider the circumstances with respect to the $\langle 310\rangle$ zone pattern. In the pattern, there is a $\{3 / 2,1 / 2,0\}$ superlattice spot between the origin and a $\{310\}$ fundamental spot. This means that the $\{310\}$ spot must be indexed as the $\{310\}$ or $\{130\}$ type but not as the $\{013\}$ or $\{031\}$ type. Therefore, the diffraction pattern must be that of the $\langle 310\rangle$ or $\langle 130\rangle$ type zones of the twelve $\langle 310\rangle$ zones. For the possible four zones, the trace of bands was analysed stereographically. All of the analyses show that the trace coincides with that of the $\{112\}$ plane with 2 in the 1 index. The $\{112\}$ planes are the twin planes in $b c c$ crystals and moreover coincide with those of the lattice invariant shear system assumed in the phenomenological theory of the $f c c$ to $b c c$ martensite transformation. In Photo. 4 (b), if [1 $1 \overline{3} 0]$ zone is adopted of the possible four $\langle 310\rangle$ zones, the twin plane becomes (112) as indicated in the micrograph, (a). The $[1 \overline{3} 0]$ is nearly parallel to $[\overline{2} 11]_{T}$ of the twinned crystal on the (112) plane and the $[\overline{2} 11]_{T}$ zone pattern is acturely observed in Photo. 4(b). The remaining $\langle 010\rangle$ zone pattern is that from the matrix $\gamma$ phase. However, these three zones are not exactly parallel with one another, that is, the $[1 \overline{3} 0]$ axis makes an angle of about $8^{\circ}$ with the $[\overline{2} 11]_{T}$ and about $18^{\circ}$ with the $[0 \overline{1} 0]$ axes. The

(11) E. J. Efsic and C. M. Wayman : Trans. Met. Soc. AIME, 239 (1967), 873.

** The rotation of the pattern due to a change of the intermediate lens current was taken into account. 


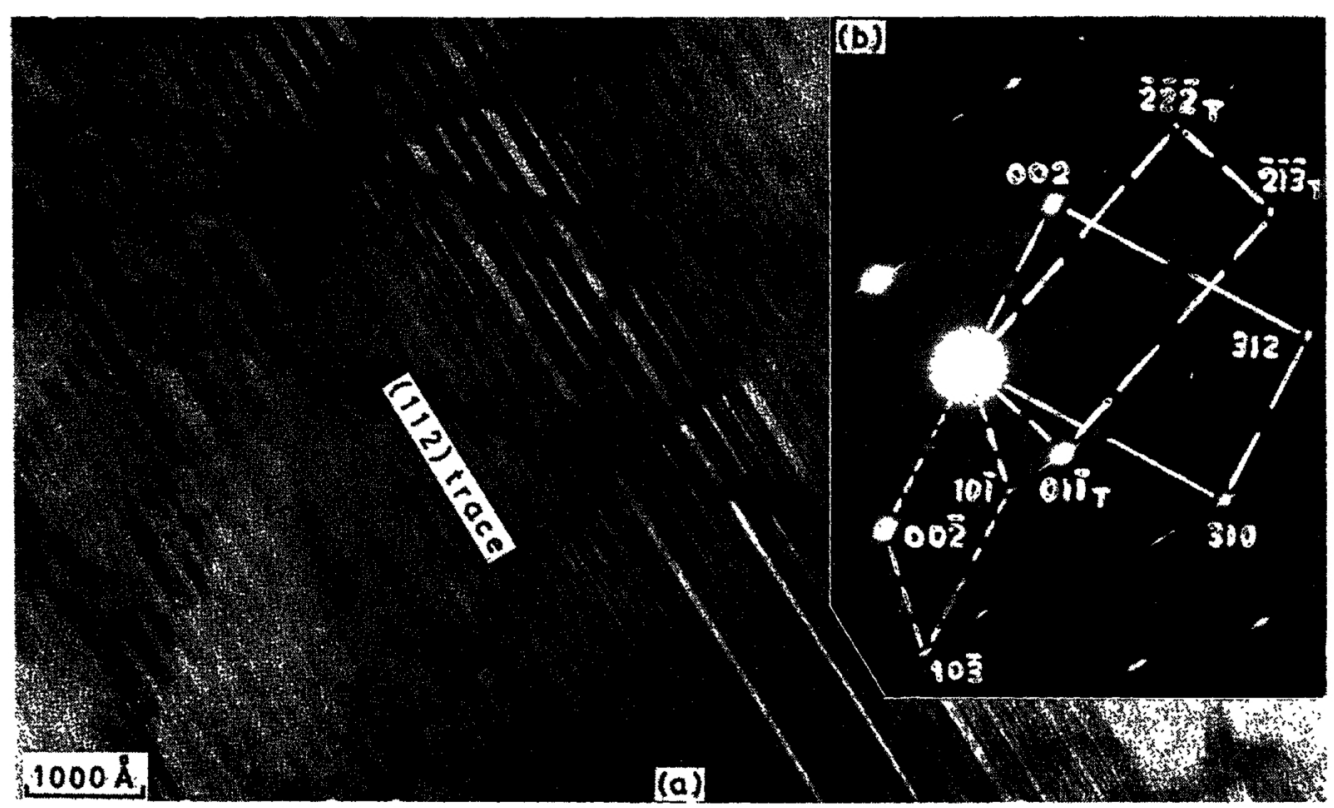

Photo. 4 (a) Transmission electron micrograph of a martensite, showing a number of transformation twins on the (112) planes

(b) Electron diffraction pattern taken from the whole area of (a)

simultaneous appearance of three zone patterns may be due to some buckling of thin specimens and so on.

A different zonal diffraction pattern was taken in order to make clear that the internal faults in the martensite are twin faults on a plane of the $\{112\}$ type, which is given in Photo. 5. As explained in the key diagram, Fig. 2, the diffraction pattern consists of two sets of reflections, both of which are identified as the $<311>$ zone's and have a twin relation with the $\{112\}$ plane. It should be noticed here that one set of the reflections have a superlattice spot and the other does not have it between the origin and the $\{110\}$ fundamental spot. In the same way as in Photo. 4 (b), we can choose [113] of possible four zones for the $\langle 311\rangle$ zone pattern indicated with a solid line. Another the $\langle 311\rangle$ zone pattern indicated with a dotted line can be formed by the twinned crystals on either of the $(2 \overline{1} 1),(\bar{I} 21)$ and (112) planes. Of these, the (112) plane must be adopted as the twin plane taking account of the lack of $(1 / 2,1 / 2$, $0)_{T}$ spot and of the direction of streaks which were observed in a diffraction pattern taken by slightly tilting the specimen. So the other $\langle 311\rangle$ zone pattern is indexed to be $[\overline{13} \overline{1}]_{T}$ as shown in the diagram. Thus, it can be concluded that the internal faults in the ordered $\mathrm{Fe}_{3} \mathrm{Pt}$ martensite are $\{112\}$ twin faults as in the case of ordinary iron alloys and steels, and moreover the twin plane has 2 in the $l$ index.

Here the reason why the transformation twins are observed on $\{112\}$ planes of $l=2$ will be discussed from the physical point of view. These $\{112\}$ planes are composed of stacking sequences of two kinds of atomic planes respectively, one of which consists of only $\mathrm{Fe}$ atoms and the other of both $\mathrm{Fe}$ and $\mathrm{Pt}$ atoms (see Fig. $3(\mathrm{a})$ ). On the other hand, the planes of $l \neq 2$, namely $\{121\}$ and $\{211\}$ type planes, are all composed of stacking sequences of atomic planes consisting of both $\mathrm{Fe}$ and $\mathrm{Pt}$ atoms (see Fig. $3(\mathrm{~b})$ ). Moreover it must be noted that

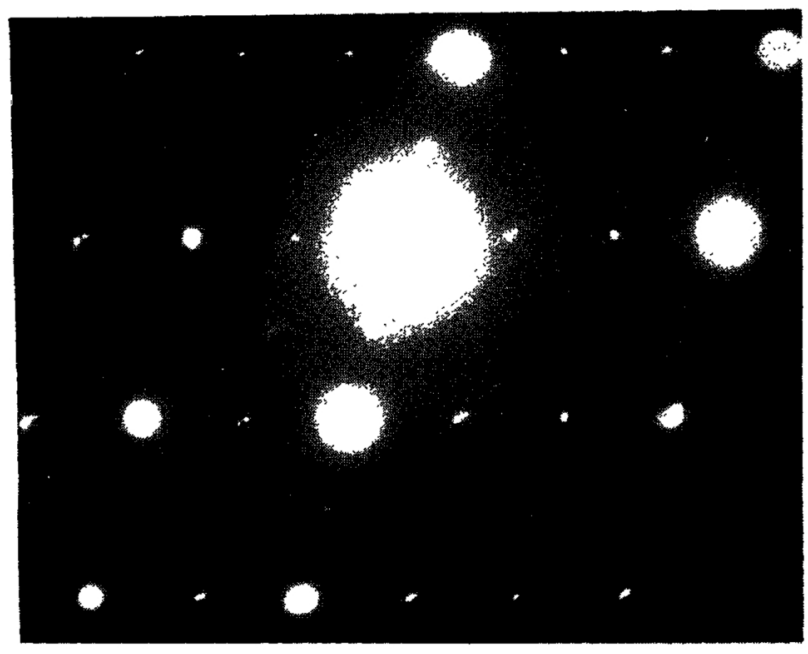

Photo. 5 Twinned diffraction pattern taken from a martensite, the electron beam being approximately parallel to the $[\overline{1} 13]$ of matrix and the $[\overline{1} \overline{3} \overline{1}]$ of twinned crystal

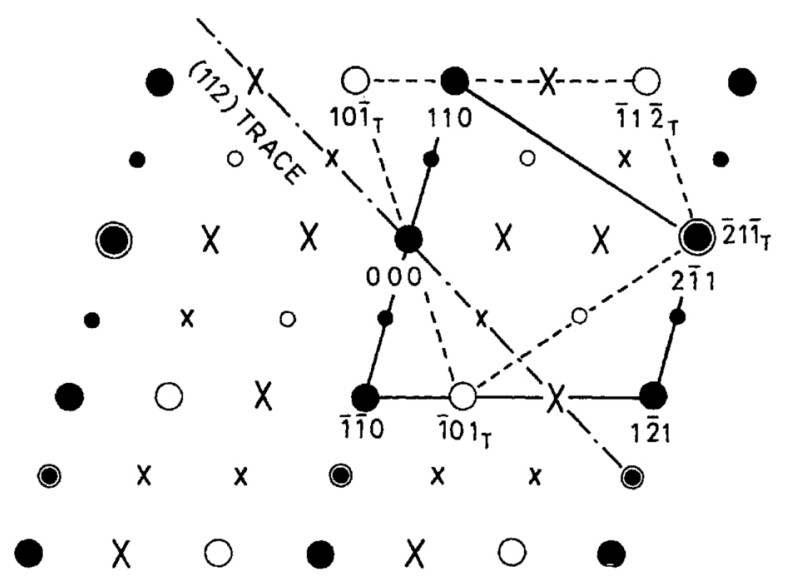

Fig. 2 Key diagram of Photo. 5. Suffix ' $T$ ' means twin spots

- : Fundamental and superlattice spots of matrix

$\bigcirc$ : Fundamental and superlattice spots of twin

$\times \times$ : Spots due to double reflection of the fundamental and superlattice spots, respectively 
the ordered structures formed by twinning shears on $\{112\}$ planes of $\bar{l}=2$ and $l \neq 2$ are different from each other. Fig. 3 (a) shows the atomic arrangement after the twinning shear on the (112) plane, the projection plane being $(1 \overline{1} 0)$. It can be found clearly that the ordered structure in the twinned crystal is quite the same as that before thetwinning shear. On the contrary, the

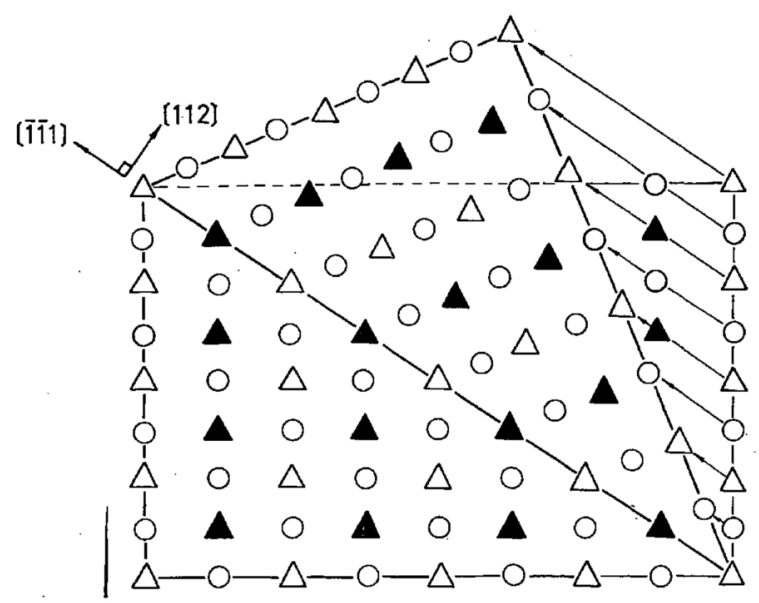

(a)

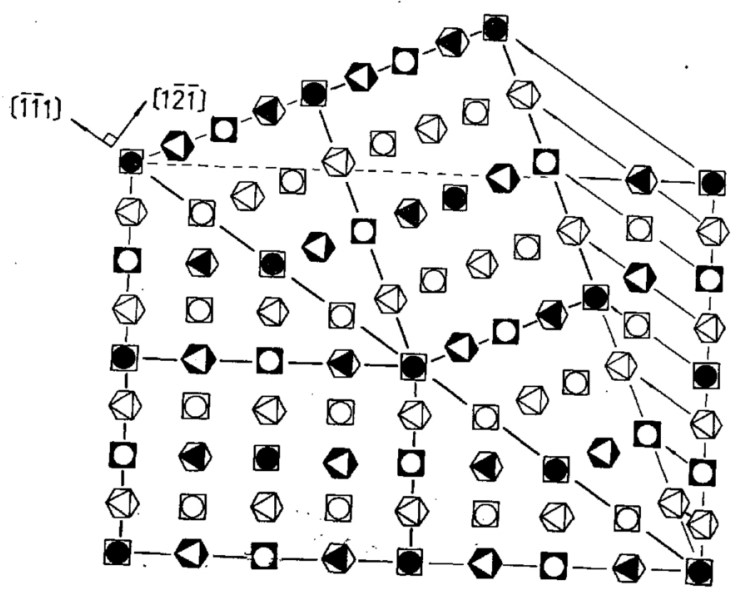

(b)

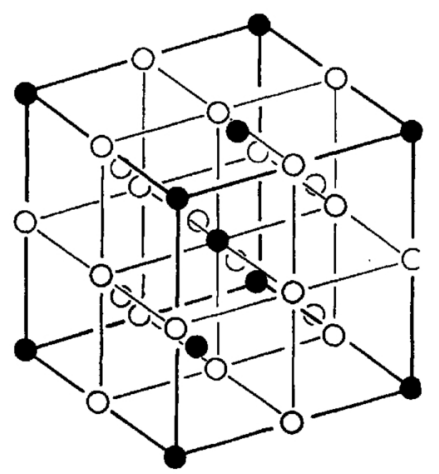

(c)

Fig. 3 (a)(b) Atomic structures subjected to twinnings in the ordered $b c c \mathrm{Fe}_{3} \mathrm{Pt}$ alloy, (a) being that due

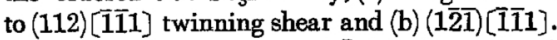
Their projection planes are (110) in (a) and (101) in (b). Circles, triangles, squares and hexagons represent atoms on the 1 st, 2 nd, 3 rd and 4 th layers, respectively, and open symbols represent $\mathrm{Fe}$ atoms and closed symbols $\mathrm{Pt}$ atoms

(c) An ordered bcc unit cell obtained by twinning on $(\mathbf{1} \overline{2} \overline{1})$

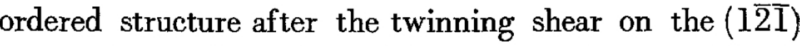
plane changes from the original one as shown in Fig. 3 (b), the projection plane of which is the $(\overline{1} 0 \overline{1})$ plane. Fig. 3 (c) shows the unit cell of the ordered $b c c$ structure obtained by the twinning shear on the $(1 \overline{2} \overline{1})$, which differs from that of Fig. $1(b)$. Since the change of the ordered structure would require large energies, the twinning shear on the $(1 \overline{2} \bar{I})$ plane may be difficult to occur, compared with that on the (112) plane.

\section{(3) Habit plane}

The habit plane measurements were carried out for martensite plates which are small in size and have comparatively straight boundaries. An example of such martensites is shown in Photo. 6(a). In the martensite, fine striations due to transformation twins are also seen though their contrast is very faint. The striations extend all the way from one interface to the other, and then the martensite does not exhibit a mid-rib region as in the case of $\mathrm{Fe}-\mathrm{Ni}$ alloys ${ }^{(12)}$.

In Photo. 6 (a), the boundary trace was analysed with respect to the diffraction pattern, (b), taken from the

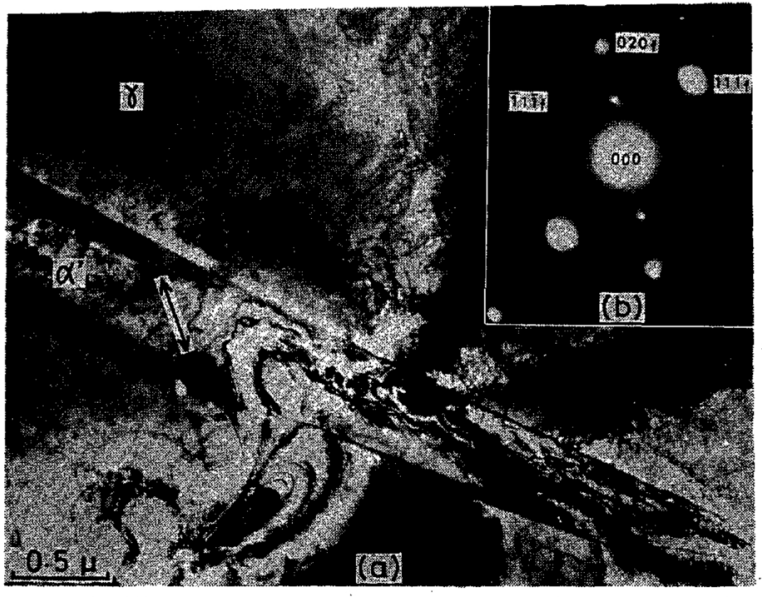

Photo. 6 (a) Transmission electron micrograph of a martensite plate used for the habit plane determination, showing the straight boundaries and fine striations in the martensite due to twin faults $(\leftrightarrow)$

(b) Electron diffraction pattern from the surrounding matrix $r$ phase in the (101) orientation

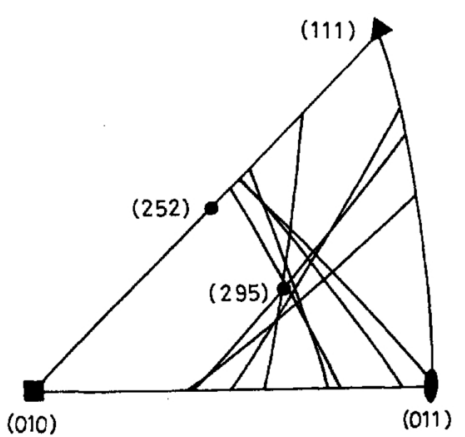

Fig. 4 Stereographic one-face trace analysis of the habit planes of eight martensite plates in an $\mathrm{Fe}_{3} \mathrm{Pt}$ alloy

(12) K. Shimizu : J. Phys. Soc. Japan, 17(1962), 508. 
region of the surrounding austenite. The same analyses were done for eight martensites, and their results are shown stereographically in Fig. 4. In the figure, eight arcs are parts of the great circles of zone planes containing the boundary traces of eight martensites. This stereograph shows that the habit plane of the ordered $\mathrm{Fe}_{3} \mathrm{Pt}$ martensite is near $\{295\}$ rather than $\{252\}$. The recult is consistent with that reported by Efsic and Wayman for the disordered $\mathrm{Fe}-\mathrm{Pt}(3: 1)$ martensite.

\section{(4) Orientation relationship}

Photos. 7 (a) and (b) are electron diffraction patterns taken from the areas containing both martensite and austenite crystals, from which the orientation relationship between martensite and austenite was determined. Two sets of reflections in (a) are a [101] zone pattern for the matrix and a [II1] zone pattern for the martensite. From the pattern, it can be recognized that the (111) plane of matrix is parallel to the (011) plane of the martensite. However, the parallelism between their zone axes is not so accurate because of the relaxation of Laue's conditions in electron diffraction. So, another zonal diffraction pattern was taken in which the zone axes are [111] for the austenite and [011] for the martensite. (b) is the pattern, from which it can be deduced that [101] of the austenite and [I11] of the martensite make an angle of absut $3.5^{\circ}$ to each other. Thus, the orientation relationship can be represented as follows :

$$
\begin{aligned}
& (111)_{f} / /(011)_{b^{\prime}} \\
& {[101]_{f} 3.5^{\circ} \text { apart from }[\text { III }]_{b^{\prime}} .}
\end{aligned}
$$

This represents roughly the Greninger-Troiano relationship and is consistent with that obtained by Efsic and Wayman using an X-ray technique.

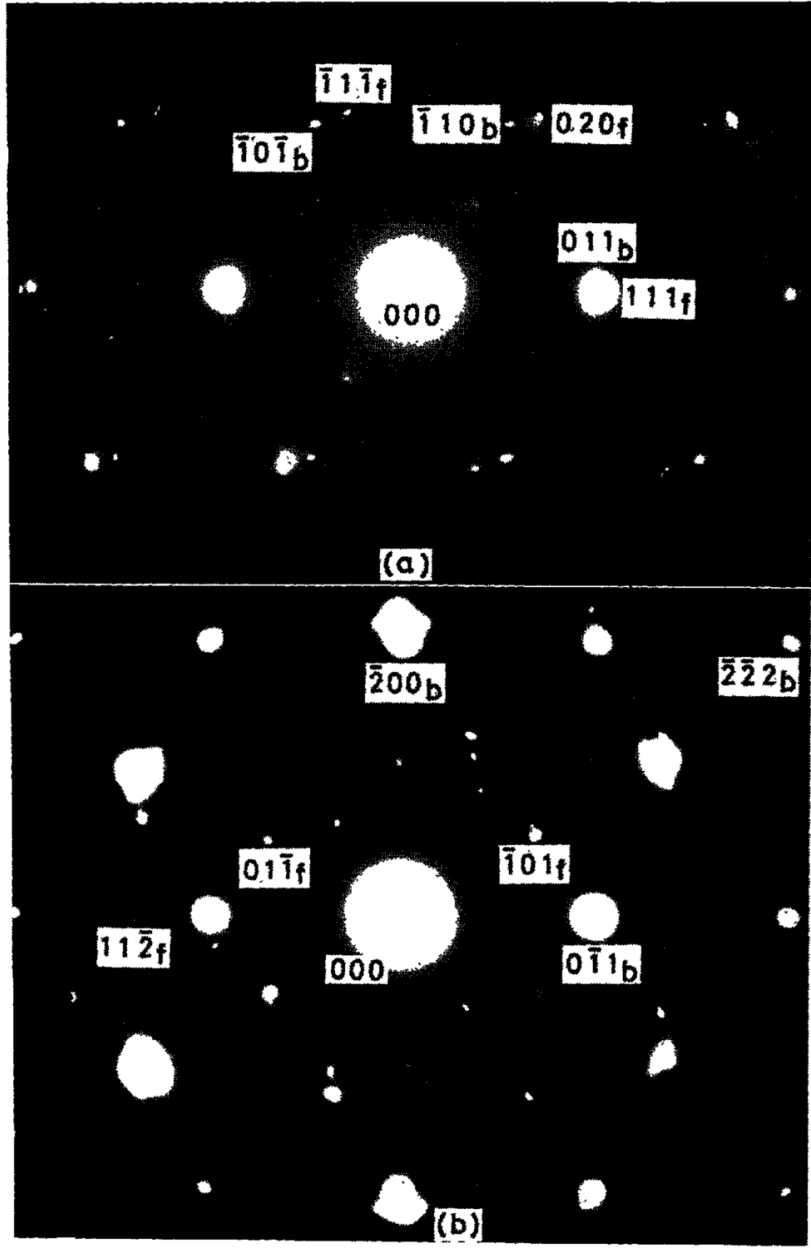

Photo. 7 Electron diffraction patterns showing the orientation relationship between the matrix and the martensite. The electron beams are nearly parallel to $[\overline{1} 01]_{f},[\overline{1} \overline{1} 1]_{b}$ in $(a)$ and to $\{\overline{1} \overline{1} \overline{1}\}_{f},[0 \overline{1} \overline{1}]_{b}$ in $(b)$.

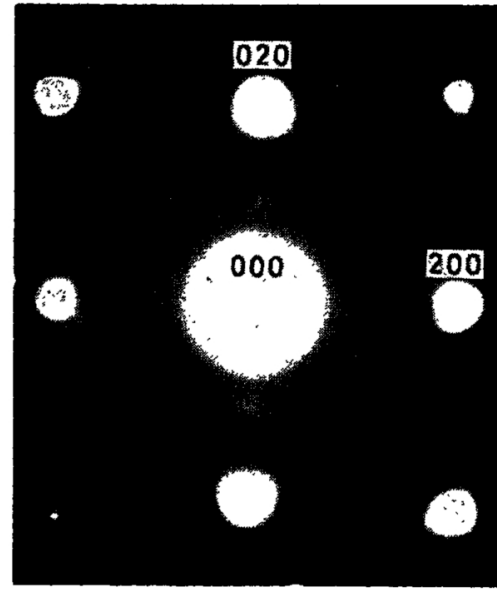

(a)

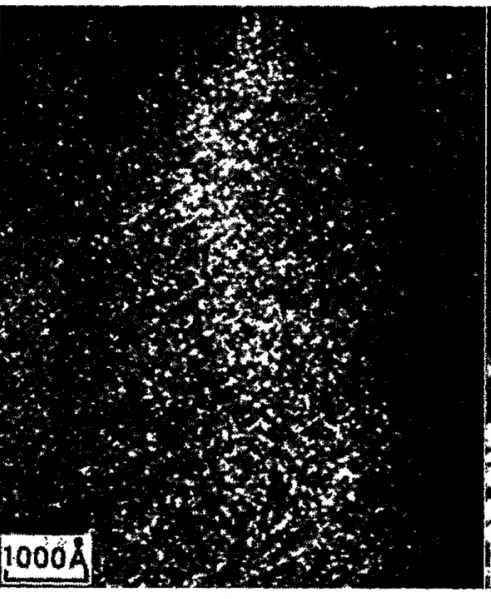

(b)

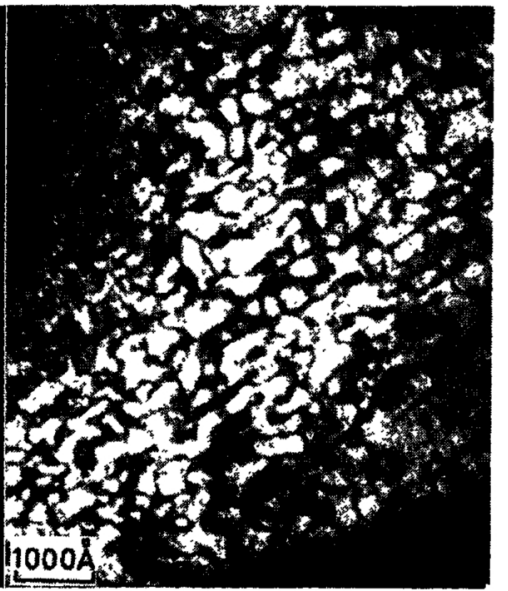

(c)

Photo. 8 (a) Electron diffraction pattern taken from the matrix $\gamma$ phase in the (001) orientation of the specimen quenched from $1000^{\circ} \mathrm{C}$

(b) (c) Dark field images formed by the (100) superlattice reflection of the matrix $\gamma$ phase in (001) orientation of the specimens annealed at $650^{\circ} \mathrm{C}$ for $0.5 \mathrm{hr}$ in (b) and $24 \mathrm{hr}$ in (c), respectively

\section{(5) Relation between Ms temperature and} domain size in austenite

According to the phase diagram of the Fe-Pt alloy system after Kussmann and Rittberg ${ }^{(10)}$, the martensitic transformation should not occur in a specimen in composition of 25 at $\% \mathrm{Pt}$ when the specimen is quenched into water at room temperature from $1000^{\circ} \mathrm{C}$. A preliminary experiment, however, showed the formation of martensite plates in the specimen of the same composition. If the specimen was annealed at $650^{\circ} \mathrm{C}$ for $0.5 \mathrm{hr}$ after the quenching from $1000^{\circ} \mathrm{C}$, then the specimen does not exhibit any martensitic transformation. These results 
rather agree with those obtained by Berkowitz et al. ${ }^{(13)}$ using X-ray and metallographic techniques.

Photo. $8(a)$ is a diffraction pattern taken from the matrix $\gamma$ phase of the specimen quenched into water from $1000^{\circ} \mathrm{C}$, which is $(001)$ zone pattern. There are seen weak and diffuse extra spots between the fundamental spots. This suggests that the as-quenched specimen is not in a completely disordered state but in a short range ordered state. Microscopic observations of such a specimen revealed $\alpha^{\prime}$ martensite plates. From the amount of martensites, the Ms temperature of the specimen can be estimated to be slightly above room temperature. (b) is a dark-field image formed by the (100) superlattice reflection of the $(001)$ zone pattern which was taken from the specimen annealed at $650^{\circ} \mathrm{C}$ for $0.5 \mathrm{hr}$ after the quenching from $1000^{\circ} \mathrm{C}$. The (100) superlattice reflection is sharper than that in (a) and correspondingly the ordered domains become visible in the dark field image, the domain size being less than $100 \AA$. Similar domains are also observed in the early stage of ordering in many other superlattice alloys. As mentioned already, the specimen annealed for $0.5 \mathrm{hr}$ at $650^{\circ} \mathrm{C}$ exhibits the martensitic transformation, provided that the specimen is subzero-cooled after the water-quenching. The Ms temperature was estimated to be about $-50^{\circ} \mathrm{C}$ from subzero temperature and the amount of martensites formed. (c) shows the domain structure in the specimen annealed for $24 \mathrm{hr}$ at $650^{\circ} \mathrm{C}$, which was obtained in the same way as (b). The size of domains is larger than those in (b) and about $500 \AA$. The specimen having this domain size does not exhibit any martensitic transformation even if it is subzero-cooled into $-196^{\circ} \mathrm{C}$, that is, the Ms temperature is below the liquid nitrogen temperature. These results suggest that the Ms temperature drops rapidly with the growth of ordered domains. This is consistent with the result of Berkowitz et al. ${ }^{(13)}$ obtained by the measurement of magnetization in 27.1 at \% $\mathrm{Pt}$ alloy.

\section{Conclusion}

An electron microscope study has been made on the retained austenite and martensite in an $\mathrm{Fe}_{3} \mathrm{Pt}$ alloy quenched from $1000^{\circ} \mathrm{C}$ followed by annealing at $650^{\circ} \mathrm{C}$ for various periods, with the results summarized as follows :

(1) The $b c c$ martensite obtained by subzero cooling was found to have a superlattice which was expected to be formed by the Bain distortion from the $\gamma$ phase.

(2) In the martensite crystals, a number of transformation twins were observed on the $\{112\}$ type planes and not on the $\{121\}$ or $\{211\}$ type planes.

(3) The habit plane of martensite plates and the orientation relationship between martensite and austenite were consistent with those determined by Efsic and Wayman using an X-ray technique for the disordered Fe-Pt alloy of the same composition, and with their calculations based on the phenomenological theory of martensitic transformation.

(4) Observing the domain structures in ordered $\gamma$ phase, it is clarified that the Ms temperature dropped rapidly according as the size of domains increased.

\section{Acknowledgments}

The authors wish to thank Emeritus Prof. Z. Nishiyama for his encouragement and discussion. This work was partly supported by the Grant-in-Aid for Fundamental Scientific Research from the Ministry of Education.

(13) A. E. Berkowitz, F. J. Donahoe, A.D. Franklin and R.P. Steijn : Acta Met., $5(1957), 1$. 